\title{
Efimov resonances above four-boson threshold
}

\author{
A. Deltuva \\ Institute of Theoretical Physics and Astronomy, Vilnius University, Saulètekio al. 3, LT-10257 Vilnius, Lithuania
}

(Received March 7, 2021)

\begin{abstract}
Four-boson Efimov physics is well known in the negative energy regime but far less above the four-body breakup threshold. The part of this region with negative two-boson scattering length is studied solving rigorous four-particle scattering equations for transition operators in the momentum space. Moving away from the unitary limit the Efimov tetramers evolve from unstable bound states into resonances. Their energies and widths are studied as functions of the two-boson scattering length; a universal behavior is established and given in a dimensionless representation. The Efimov tetramers have finite width in the whole regime; they broaden rapidly in the resonance regime but remain narrower than the associated trimer. The resonant behavior is most clearly seen in the four-particle recombination rate.
\end{abstract}

\section{INTRODUCTION}

Efimov physics in few-body systems manifests itself by a rich spectrum of bound and/or resonant states independent of the short-range interaction details. First, considering the three-body system with large two-body scattering length $a, \mathrm{~V}$. Efimov [1] predicted an infinite number of bound three-body states, called Efimov trimers, with geometric energy spectrum in the unitary limit $a \rightarrow \infty$. After this pioneering study a large number of theoretical and experimental works emerged as summarized in several review papers [2 [6], covering applications from nuclear to molecular and cold atom physics.

Beside the most extensively investigated three-body systems, also four-body systems attracted considerable interest. In particular, in the system of four identical bosons with resonant $s$-wave interactions it was found that for each Efimov trimer there exist two four-boson states [7], often called Efimov tetramers. Except for the lowest two, all the other are unstable bound states since they are above the ground state trimer. In the negative energy regime below the four-body breakup threshold the properties of the Efimov tetramers, i.e., the evolution of their energies and widths with the scattering length, have been accurately calculated. At particular positive values of the scattering length $a$ the tetramers are crossing the particle-trimer or dimer-dimer threshold and becoming inelastic virtual states [8]. On the negative $a$ side the tetramers decay through the four-particle threshold of vanishing energy $E=0$. Each intersection of the tetramer and $E=0$ threshold taking place at specific value of $a$ leads to a resonant enhancement of the four-particle recombination [9, 10].

Once the tetramers enter the positive energy regime, they become true resonances, however, their energies and widths quantitatively are not known. Their study was precluded by the complications in rigorously describing the four-particle continuum with open many-cluster channels. Typically, in the coordinate-space differential equation approach complicated boundary conditions must be imposed [11], while in the momentum-space integral equation framework one faces complicated singularities [12]. On top of that, the Efimovian character means that the problem involves the states of very different sizes and energies, that require a very careful and accurate treatment.

Recent developments in the description of four-nucleon reactions above the breakup threshold [12], especially the search of the four-neutron resonance 13], paved the way also for the study of four-boson Efimov resonances. Momentum-space integral equations for transition operators with complex-energy and special weight method for the singularity treatment is expected to provide an accurate description of the four-boson system at positive energies, and will be used in the present work, aiming to determine the properties of four-boson Efimov resonances and their impact on collision processes. The present study can also be viewed as an extension of the previous work [14] that considered the Efimov resonances in the three-boson system.

Section II shortly recalls the four-particle scattering equations and essential aspects of calculations whereas results are given in Sec. III. The summary is presented in Sec. IV.

\section{THEORY}

Resonance corresponds to the pole of the $S$-matrix or the related transition operator in the unphysical sheet of the complex-energy plane. Its location $E^{R}-i \Gamma / 2$ is determined by the real part of the energy $E^{R}$ and the width $\Gamma$. The Laurent series expansion for the dependence of the transition operator on the energy $E$ is led by the pole term proportional to $1 /\left(E-E^{R}+i \Gamma / 2\right)$, but higher-order background terms contribute as well. As long as $\Gamma$ is not too large, the pole is not too far from the real energy axis and therefore affects physical processes in the system at energies around $E^{R}$. Under these conditions the resonance parameters can be determined from the energy dependence of the transition operator in the physical region, i.e., from the physical observables. This procedure was used for the search of the four-neutron resonance [13] and Efimov three-boson resonances [14]; it is described in more details in Refs. [13, 14] and is used also in the present work. 
The study of the four-neutron resonance [13] considered $4 \rightarrow 4$ transition operator. It could be used also here, however, a more practical choice is a subset of twocluster transition operators, that are more directly related to amplitudes for boson-trimer elastic and inelastic scattering or four-boson recombination. These transition operators $\mathcal{U}_{\alpha \beta}(Z)$ are obtained solving symmetrized form of equations originally proposed by Alt, Grassberger, and Sandhas (AGS) [15], i.e.,

$\begin{aligned} \mathcal{U}_{11}(Z)= & P_{34}\left(G_{0} t G_{0}\right)^{-1}+P_{34} U_{1} G_{0} t G_{0} \mathcal{U}_{11}(Z) \\ & +U_{2} G_{0} t G_{0} \mathcal{U}_{21}(Z) \\ \mathcal{U}_{21}(Z)= & \left(1+P_{34}\right)\left(G_{0} t G_{0}\right)^{-1}+\left(1+P_{34}\right) U_{1} G_{0} t G_{0} \mathcal{U}_{11}(Z)\end{aligned}$

where the subscripts $\alpha, \beta=1$ (2) denote the $3+1(2+2)$ clustering, $G_{0}=\left(Z-H_{0}\right)^{-1}$ is the free four-boson resolvent of the four-particle system with kinetic energy operator $H_{0}$ and $Z=E+i 0, t=v+v G_{0} t$ is the two-boson transition matrix derived from the pair (12) potential $v$, and

$$
U_{\alpha}=P_{\alpha} G_{0}^{-1}+P_{\alpha} t G_{0} U_{\alpha}
$$

are the $3+1$ and $2+2$ subsystem transition operators. $G_{0}, t$, and $U_{\alpha}$ depend on the complex energy parameter $Z$, but for brevity this dependence is suppressed in the notation. The bosonic symmetry in the fourbody system is imposed by permutation operators $P_{34}$, $P_{1}=P_{12} P_{23}+P_{13} P_{23}$, and $P_{2}=P_{13} P_{24}$ where $P_{a b}$ interchanges particles $a$ and $b$.

Symmetrized AGS equations (11) are solved in the momentum-space partial-wave representation $\left|k_{x} k_{y} k_{z}\left[\left(l_{x} l_{y}\right) J l_{z}\right] \mathcal{J} \mathcal{M}\right\rangle_{\alpha}$, where $k_{x}, k_{y}, k_{z}$ are the magnitudes of the Jacobi momenta [10] and $l_{x}, l_{y}, l_{z}$ are the associated orbital angular momenta, that are coupled via the intermediate subsystem angular momentum $J$ to the total four-body angular momentum $\mathcal{J}$ with the projection $\mathcal{M}$. In the context of Efimov tetramers $\mathcal{J}=0$. Furthermore, given the universality, for the numerical efficiency it is convenient to restrict the two-body interaction to the $s$-wave, i.e., $l_{x}=0$, and to take it of a separable form

$\left\langle k_{x}^{\prime} l_{x}|v| k_{x} l_{x}\right\rangle=\delta_{l_{x} 0} e^{-\left(k_{x}^{\prime} / \Lambda\right)^{2}} \frac{2}{\pi m}\left\{\frac{1}{a}-\frac{\Lambda}{\sqrt{2 \pi}}\right\}^{-1} e^{-\left(k_{x} / \Lambda\right)^{2}}$,

where $m$ is the boson mass and $\Lambda$ the momentum cutoff parameter. With these constrains $l_{y}=l_{z}=J$ and formally is unlimited from above, however, practical calculations reveal that $l_{y}, l_{z}, J \leq 1$ is sufficient for an accuracy better than 1\%. Although Ref. [10] included higher waves $l_{y}, l_{z}, J \leq 2$ and thereby achieved accuracy better than $0.1 \%$, the corresponding extension in the present work would not significantly improve the extraction of resonance parameters, since they have larger error bars due to other reasons as shown and discussed in the next section.
In the region of negative two-boson scattering length $a$ there are no bound dimers, and the singularities in the kernel of the AGS equations (11) arise due to the Efimov trimer bound state poles in $U_{1}$ and due to free resolvent $G_{0}$. Their treatment using the complex-energy method with special integration weights is taken over from Ref. [12]. After the discretization of momentum variables the system of integral equations (1) becomes a system of linear algebraic equations. Since the spectrum of the four-boson system near unitarity is more rich compared to the four-nucleon system, it is advisable to solve the linear system by a direct matrix inversion as in other four-boson studies [8, 10] based on the integral equations for transition operators. This is possible taking advantage of a simple potential form (3) that allows to reduce the number of continuous variables in the AGS equations (1). References [8, 10] provide more details and also relations of transition operators $\mathcal{U}_{\alpha \beta}(Z)$ to scattering amplitudes and observables.

\section{RESULTS}

The present work studies the evolution of four-boson Efimov resonances in the $a<0$ two-boson scattering length region above the four-body breakup threshold. For this purpose the scattering length $a$ is varied in Eq. (3); $|a|$ decreases when decreasing the two-boson attraction and the system moves away from the unitary limit. Special value of $a$ where the $n$-th Efimov trimer crosses the $E=0$ threshold is denoted by $a_{n}^{-}$, with $n=0$ labeling the ground state and $n \geq 1$ the excited states. In the bound state regime there are two Efimov tetramers associated with each trimer, they are labeled with two integer numbers $n, k$, where $k=1$ (2) corresponds to a more (less) tightly bound tetramer, called also deep (shallow) tetramer in the literature. Their intersections with the $E=0$ threshold are labeled by $a_{n, k}^{-}$. Although in the resonance regime the wording deep or shallow is not really meaningful, it will be employed nevertheless in order to relate the resonances to the states from which they evolved. In the unitary limit $a_{n+1}^{-} / a_{n}^{-} \approx 22.694$, the universal Efimov ratio [2]. Furthermore, $a_{n, 1}^{-} / a_{n}^{-}=$ $0.4254(2)$ and $a_{n, 2}^{-} / a_{n}^{-}=0.9125(2)$ as determined in accurate numerical calculations in Ref. [10]. In fact, already for $n=2$ with the force model of Eq. (3) the deviations from the above ratios are well below $0.4 \%$ as shown in Ref. [10]. For a fixed $n$ the error in $a_{n, k}^{-}$due to the limitation $l_{y}, l_{z}, J \leq 1$ is even smaller, well below $0.1 \%$. Together with the convergence study in Refs. [8, 14] this suggests that $n=2$ and $l_{y}, l_{z}, J \leq 1$ is sufficient for the extraction of the universal results with a good accuracy, better than 1\%. A further justification is provided by the fact that in this regime the two-boson effective range $r_{s}$ is already much smaller than $a$, the ratio $\left|r_{s} / a\right|$ that quantifies the finite-range corrections being of the order of 0.001 for $n=2$. For comparison, in the regime rele- 


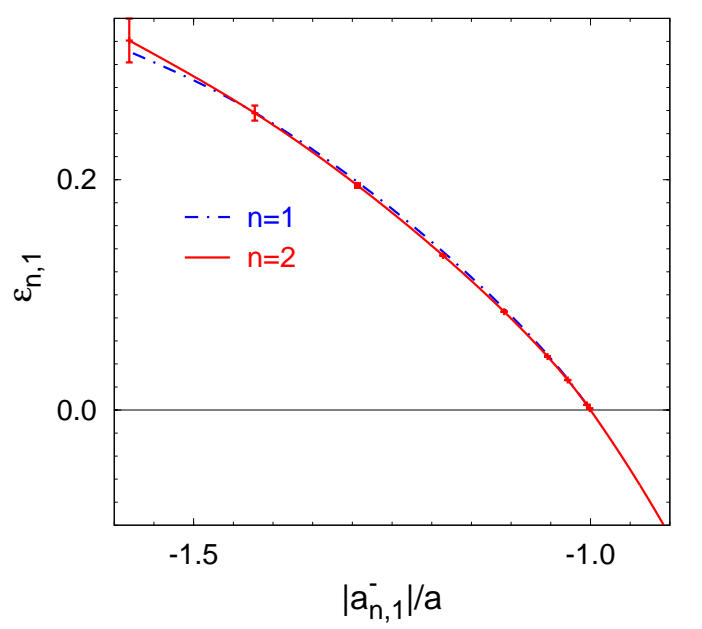

FIG. 1. (Color online) Dimensionless real energy part of the deep $(k=1)$ Efimov tetramer as a function of the inverse twoboson scattering length. The results for the states associated with the first and second excited Efimov trimer are displayed by dashed-dotted and solid curves, respectively.

vant for the $n=1$ states $\left|r_{s} / a\right|$ is of the order of 0.02 . Alternatively, large values of $|a \Lambda|$ also show that $a$ largely exceeds the interaction range; $1020<|a \Lambda|<3776$ in the present calculations for $n=2$.

The universal character of the results becomes more evident when represented in dimensionless quantities. The reference point in $a$ for each Efimov tetramer is chosen as $a=a_{n, k}^{-}$that connects the unstable bound state and resonance regimes. Preserving the consistency with the standard representation of the Efimov physics in terms of $1 / a$, the results will be given as functions of the dimensionless ratio $\left|a_{n, k}^{-}\right| / a$, with $\left|a_{n, k}^{-}\right| / a>-1$ corresponding to the unstable bound state while $\left|a_{n, k}^{-}\right| / a<-1$ in the resonance regime. Furthermore, the energy $E_{n, k}^{R}$ and width $\Gamma_{n, k}$ of the $(n, k)$-th Efimov tetramer will be presented in the dimensionless forms $\varepsilon_{n, k}=E_{n, k}^{R} m\left(a_{n, k}^{-}\right)^{2} / \hbar^{2}$ and $\gamma_{n, k}=\Gamma_{n, k} m\left(a_{n, k}^{-}\right)^{2} / \hbar^{2}$. Note that, up to the finiterange corrections, $-\hbar^{2} / m\left(a_{n, k}^{-}\right)^{2}$ is the energy of the virtual two-boson state at the crossing point $a=a_{n, k}^{-}$. Thus, $\varepsilon_{n, k}\left(\gamma_{n, k}\right)$ is the energy (width) of the $(n, k)$-th Efimov tetramer in units of the dimer virtual state energy taken at the reference point $a=a_{n, k}^{-}$.

The dimensionless energies $\varepsilon_{n, k}$ of the tetramers associated with the first two excited Efimov trimers $n=1$ and 2 are shown in Figs. 1 1and2 for the deep and shallow levels, respectively. For $n=2$ also the unstable bound state regime is partially given. In this representation both types of tetramers exhibit a qualitatively similar dependence on the scattering length: when moving away from the unitary limit, i.e., weakening the attraction between bosons, the unstable bound state turns into a resonance whose energy increases but with some saturation. The finite-range effects appear to be small also for the

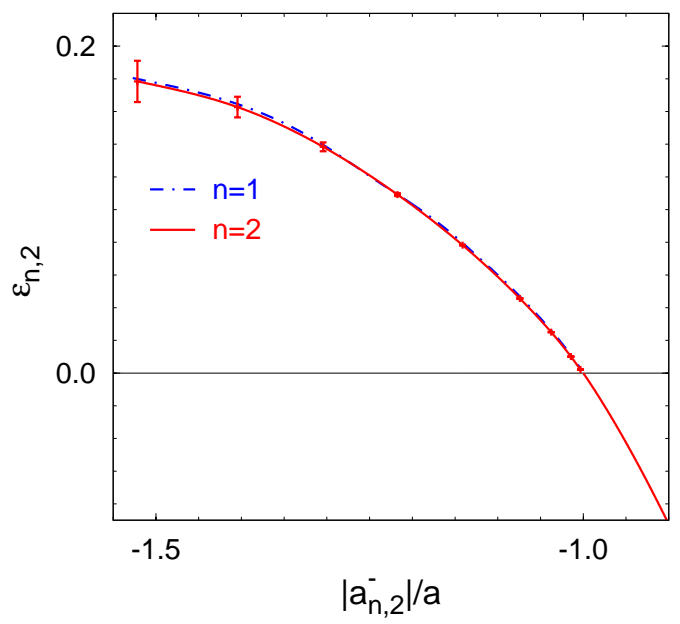

FIG. 2. (Color online) Dimensionless real energy part of the shallow $(k=2)$ Efimov tetramer as a function of the inverse two-boson scattering length. Curves are as in Fig. 1.

$n=1$ level, providing confidence on the convergence towards universal limit with increasing $n$. The evolution of the corresponding dimensionless widths $\gamma_{n, k}$ is presented in Figs. 3 and 4 for the deep and shallow tetramer, respectively. Note that also in the unstable bound state regime the tetramer width is finite, though small in the absolute size; see Ref. [8] for a detailed study. As a consequence, at the transition point $a=a_{n, k}^{-}$the four-boson resonances have finite width, in contrast to the zero width in typical cases where the true bound state evolves into the resonance as, for example, the Efimov trimer does 14]. Moving deeper into the resonance region towards smaller $\left|a_{n, k}^{-}\right| / a$ values the widths of the four-boson Efimov resonances increase. Again, the evolution is qualitatively similar for deep and shallow tetramer. For both of them the differences between $n=1$ and 2 results are slightly larger than in the $\varepsilon_{n, k}$ case; this is consistent with the unstable bound state regime where the width shows somehow slower convergence with $n$ [8]. Larger finiterange effects for the width as compared to the energy are not surprising since for the width decisive are the transitions to states of a particle plus lower-lying trimer that obviously are more affected by range corrections.

The symbols in Figs. 1 - 4 show $n=2$ results with theoretical error bars (for $n=1$ the error bars are of the same size), estimated in the same way as in previous works [13, 14]. For narrow and well pronounced resonances close to $\left|a_{n, k}^{-}\right| / a=-1$ the errors are very small. However, as the width of the resonance increases, the nonresonant background terms in the transition operators become dominant, the resonant behavior can hardly be seen, which results in large uncertainties in the determination of resonance parameters. For this reason the present results are limited to $\left|a_{n, k}^{-}\right| / a>-1.6$, however, beyond this limit the resonant behavior in the four-boson continuum is disappearing and becomes physically unob- 


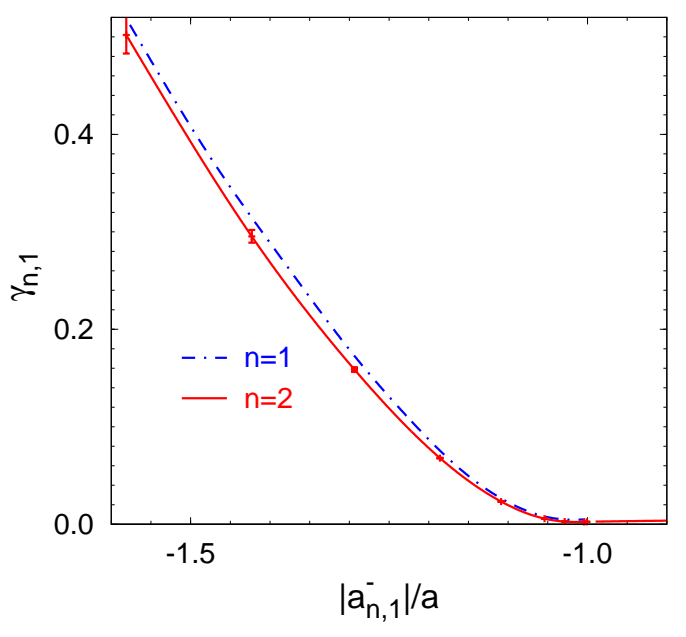

FIG. 3. (Color online) Dimensionless width of the deep $(k=$ 1) Efimov tetramer as a function of the inverse two-boson scattering length. Curves are as in Fig. 1 .

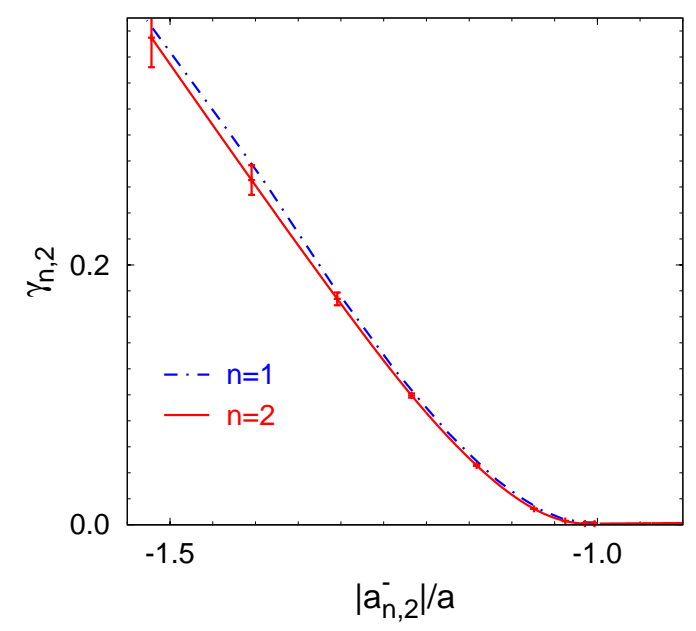

FIG. 4. (Color online) Dimensionless width of the shallow $(k=2)$ Efimov tetramer as a function of the inverse twoboson scattering length. Curves are as in Fig. 1

servable.

Given the proximity of $a_{n}^{-}$and $a_{n, 2}^{-}$, the three-boson Efimov resonances appear in a nearby regime. It is interesting to compare three- and four-boson Efimov resonances using the same scale. For this purpose $\varepsilon_{n}^{k}=$ $E_{n, k}^{R} m\left(a_{n}^{-}\right)^{2} / \hbar^{2}$ and $\gamma_{n}^{k}=\Gamma_{n, k} m\left(a_{n}^{-}\right)^{2} / \hbar^{2}$ are introduced, where $k=3$ formally corresponds to the trimer; in this case the results are taken from Ref. [14]. In other words, $\varepsilon_{n}^{k}$ and $\gamma_{n}^{k}$ are energies and widths of the respective few-boson Efimov states in units of the dimer virtual state energy taken at the common reference point $a=a_{n}^{-}$ where the trimer becomes unbound. The results for the trimer and tetramer energies are displayed in Fig. 5 by solid, dashed-dotted, and dashed curves for $k=1,2$, and 3 , respectively. To reflect the width of each state, the

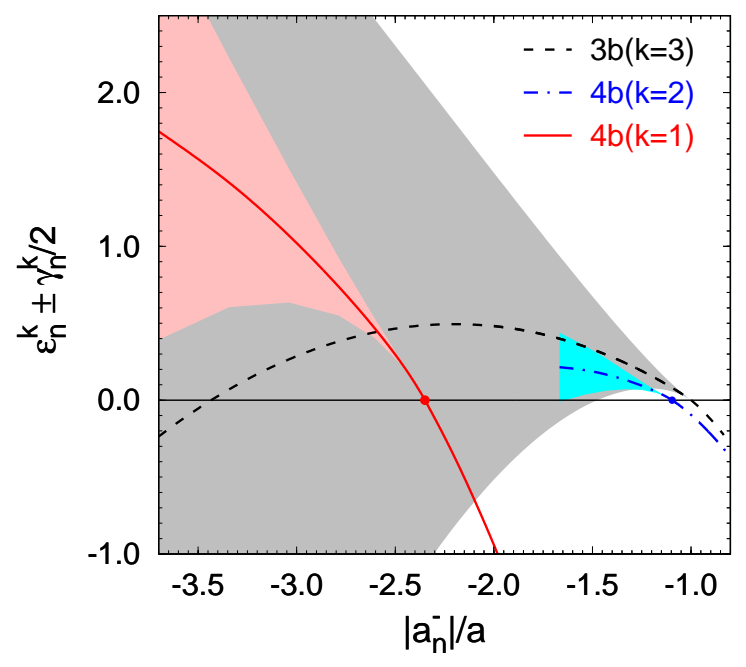

FIG. 5. (Color online) Energies of three- and four-boson Efimov resonances as functions of the inverse two-boson scattering length. The curves represent the $\varepsilon_{n}^{k}$ values while the shaded areas around each curve extend from $\varepsilon_{n}^{k}-\gamma_{n}^{k} / 2$ to $\varepsilon_{n}^{k}+\gamma_{n}^{k} / 2$ and thereby reflect the width of the respective resonances. The two points at the threshold correspond to $a=a_{n, k}^{-}$with $k=1$ (left) and 2 (right), respectively.

shaded areas around each curve cover the values between $\varepsilon_{n}^{k}-\gamma_{n}^{k} / 2$ and $\varepsilon_{n}^{k}+\gamma_{n}^{k} / 2$. The three-boson resonance broadens most rapidly and evolves into a physically unobservable subthreshold resonance with $\varepsilon_{n}^{3}<0$ residing in the third quadrant of the complex energy plane. The trajectory of the shallow tetramer is roughly parallel to the trimer, just shifted to more negative $\left|a_{n}^{-}\right| / a$ values and with lower energy and width. The complexity of four-body calculations precludes a reliable parameter extraction of very broad and practically unobservable resonances, but the similarity to the trimer case suggests the conjecture that the $k=2$ tetramer evolves into the subthreshold resonance as well when moving away from the unitary limit. Note that this is a typical behavior of resonances observed in a number of other few-body systems [16 20]. The deep tetramer evolves into a resonance with the highest energy, exceeding also the trimer. However, one has to keep in mind that there is no real crossing of these states since, given very different widths, in the complex energy plane they are far from each other; the trimer is very broad and does not affects the physical four-boson processes near the $a \approx a_{n, 1}^{-}$regime.

The present method for determining the resonance parameters from the energy dependence of physical transition operators (scattering amplitudes) is not applicable to physically unobservable very broad or subthreshold resonances, in contrast to some methods based on the complex scaling or analytic continuation in the coupling constant [17, 19]. However, it has an advantage of predicting simultaneously also the scattering observables, including both resonant contributions and nonresonant background, whose relative importance determines 


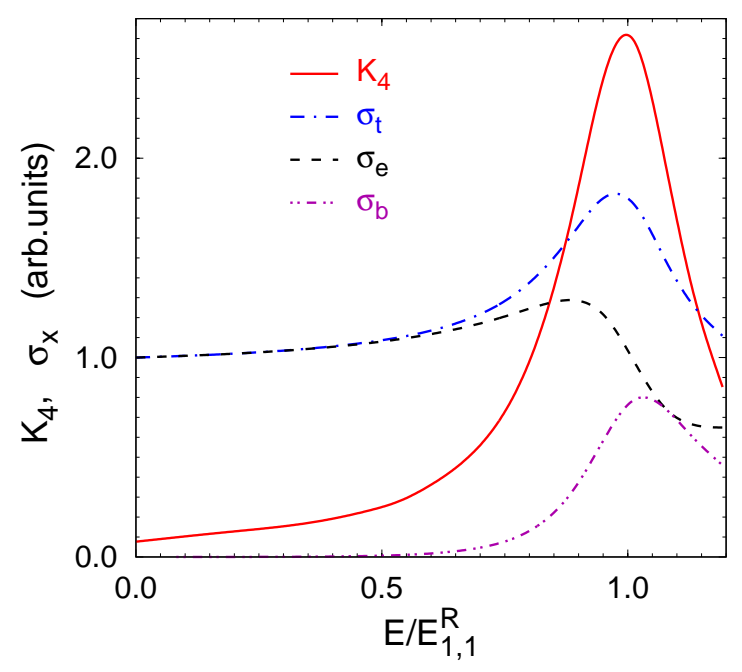

FIG. 6. (Color online) Four-boson recombination rate $K_{4}$ and cross section components for the boson-trimer scattering as functions of the system energy. The resonance corresponds to $(n, k)=(1,1)$ with $\left|a_{1,1}^{-}\right| / a=-1.1035, \varepsilon_{1,1}=0.433$, and $\gamma_{1,1} / \varepsilon_{1,1}=0.286$.

to what extent the resonant behavior is pronounced and can be observed. Figure [6 presents an example for $(n, k)=(1,1)$ at $a=0.4 a_{1}^{-}=0.9062 a_{1,1}^{-}$. Several collision channels are possible: (i) four free bosons can recombine into the ground-state Efimov trimer plus boson; (ii) a boson may scatter from the ground-state Efimov trimer elastically or (iii) inelastically, i.e., leading to the trimer breakup into three free bosons. Note that (i) is the time reverse of (iii). The characteristic observables, i.e., the four-particle recombination rate $K_{4}$ [10] and the elastic $\sigma_{e}$, breakup $\sigma_{b}$, and total $\sigma_{t}=\sigma_{e}+\sigma_{b}$ bosontrimer scattering cross sections are shown as functions of the energy. Noteworthy, although the resonance energy and width extracted from different amplitudes agree very well, the relative importance of resonant and background terms is very different for different reactions as Fig. 6 demonstrates; the resonance is most clearly pronounced in the four-boson recombination rate $K_{4}$.

\section{SUMMARY}

The four-boson continuum was studied at negative values of the two-boson scattering length. Reducing its magnitude and thereby moving away from the unitary limit, the Efimov tetramers evolve from unstable bound states into resonances. Exact scattering equations for the four-particle transition operators were solved using the momentum-space techniques. The energy dependence of various scattering amplitudes was explored to determine resonance positions and widths.

Using a simple rank-one separable potential, the tetramers associated with the first two excited Efimov trimers were considered. It was demonstrated that in a proper dimensionless representation the results for the two levels are close. Taking into account the convergence rate for a number of other four-boson quantities it was argued that $n=2$ level results approximate well the universal limit, the remaining finite-range corrections being well below $1 \%$.

In contrast to the Efimov trimers, even at the transition points $a=a_{n, k}^{-}$the tetramers (except for $n=0$ ) have finite width. However, the width reaches much higher values when going deeper into the resonance regime. Nevertheless, the tetramers broaden less rapidly than the trimer. The observed resonance evolution, when reducing the two-boson attraction and therefore $|a|$, is typical also in many other systems: the real energy part rises with the decreasing rate while the width rises with the increasing rate, until the resonance becomes very broad, physically unobservable, and cannot be followed anymore by the present method. The seen similarity between the trimer and shallow tetramer evolution suggests that also the latter evolves into a physically unobservable subthreshold resonance. In the physically observable regime the shallow tetramer is lower in energy than the trimer while the deep tetramer can rise to higher energy values.

The advantage of the transition operator method is the ability to predict the physical observables. Presented examples demonstrate that the relative importance of resonant and background contributions depends strongly on the considered observable, and suggest that the fourparticle recombination rate exhibits the most pronounced resonant behavior.

The author acknowledges support by the Alexander von Humboldt Foundation under grant no. LTU1185721-HFST-E.
[1] V. Efimov, Phys. Lett. B 33, 563 (1970).

[2] E. Braaten and H.-W. Hammer, Phys. Rep. 428, 259 (2006).

[3] H. W. Hammer and L. Platter, Annu. Rev. Nucl. Part. Sci. 60, 207 (2010).

[4] P. Naidon and S. Endo, Reports on Progress in Physics
80, 056001 (2017).

[5] C. H. Greene, P. Giannakeas, and J. Perez-Rios, Rev. Mod. Phys. 89, 035006 (2017).

[6] A. Kievsky, L. Girlanda, M. Gattobigio, and M. Viviani, arXiv:2102.13504 (2021).

[7] H. W. Hammer and L. Platter, Eur. Phys. J. A 32, 113 
(2007).

[8] A. Deltuva, Few-Body Syst. 54, 569 (2013).

[9] J. von Stecher, J. P. D'Incao, and C. H. Greene, Nature Phys. 5, 417 (2009).

[10] A. Deltuva, Phys. Rev. A 85, 012708 (2012).

[11] R. Lazauskas, Phys. Rev. C 91, 041001(R) (2015).

[12] A. Deltuva and A. C. Fonseca, Phys. Rev. C 86, 011001(R) (2012).

[13] A. Deltuva, Phys. Lett. B 782, 238 (2018).

[14] A. Deltuva, Phys. Rev. C 102, 034003 (2020).

[15] P. Grassberger and W. Sandhas, Nucl. Phys. B2, 181 (1967); E. O. Alt, P. Grassberger, and W. Sandhas, JINR report No. E4-6688 (1972).

[16] A. Hemmdan, W. Glöckle, and H. Kamada, Phys. Rev. C 66, 054001 (2002).

[17] R. Lazauskas and J. Carbonell, Phys. Rev. C 71, 044004 (2005).

[18] F. Bringas, M. T. Yamashita, and T. Frederico, Phys. Rev. A 69, 040702(R) (2004).

[19] R. Lazauskas and J. Carbonell, Phys. Rev. C 72, 034003 (2005).

[20] T. Hyodo, T. Hatsuda, and Y. Nishida, Phys. Rev. C 89, 032201(R) (2014). 